\title{
Preparation and Characterization of Hematite-Multiwall Carbon Nanotubes Nanocomposite
}

\author{
M. Krajewski • A. Malolepszy • L. Stobinski • \\ S. Lewinska • A. Slawska-Waniewska $\cdot$ M. Tokarczyk • \\ G. Kowalski · J. Borysiuk · D. Wasik
}

Received: 7 June 2014 / Accepted: 12 September 2014 / Published online: 16 October 2014

(C) The Author(s) 2014. This article is published with open access at Springerlink.com

\begin{abstract}
The aim of this work is to study the preparation and characterization of a new nanocomposite which consists of chemically-modified multiwall carbon nanotubes covered by randomly-deposited nanoparticles of hematite. The morphology, structural and physical properties of the investigated nanomaterial were determined by means of transmission electron microscopy, X-ray diffraction and vibrating sample magnetometry at ambient conditions. The presence of residual catalyst nanospheres inside multiwall carbon nanotubes was confirmed by transmission electron microscopy. The signal coming from this contamination was under the detection limit of X-ray diffractometer, therefore it was not registered.
\end{abstract}

Keywords Hematite Multiwall carbon nanotubes . CVD $\cdot$ TEM $\cdot$ XRD $\cdot$ VSM

M. Krajewski (西) · M. Tokarczyk · G. Kowalski · J. Borysiuk · D. Wasik

Faculty of Physics, Institute of Experimental Physics,

University of Warsaw, Hoza St.69, 00-681 Warsaw, Poland

e-mail: marcin.krajewski@fuw.edu.pl

A. Malolepszy

Faculty of Materials Science and Engineering, Warsaw University of Technology, Woloska St. 141, 02-507 Warsaw, Poland

\section{Stobinski}

Institute of Physical Chemistry, Polish Academy of Sciences, Kasprzaka St. 44/52, 01-224 Warsaw, Poland

L. Stobinski

University Research Centre "Functional Materials", Warsaw

University of Technology, Woloska St. 141, 02-507 Warsaw, Poland

S. Lewinska · A. Slawska-Waniewska · J. Borysiuk Institute of Physics, Polish Academy of Sciences, Al. Lotnikow 32/46, 02-668 Warsaw, Poland

\section{Introduction}

In 1991, Iijima managed to prepare the first multiwall carbon nanotubes (MWCNTs) via arc discharge process [1]. Since then, carbon nanotubes (CNTs) became the source of advanced studies in the field of physics, chemistry and material sciences. At the same time, CNTs started to play a significant role in numerous applications in many fields including: medicine [2], high-performance adsorbents [3], sector of energy storage and conversion $[4,5]$, etc.

There are four commonly-used CNTs fabrication techniques: arc discharge [1], laser ablation [6], chemical vapor deposition (CVD) [7] and vapor-liquid-solid (VLS) method [8]. However, the two latter techniques seem to have certain advantages over the other. For instance, they are not high temperature processes. The growth and dimensions of CNTs (via CVD or VLS) can be easily-controlled by fitting reaction parameters such as: temperature, source of carbon, or catalyst concentration. However, CNTs growth complications arise when using metallic catalysts such as iron $(\mathrm{Fe})$, cobalt $(\mathrm{Co})$ or nickel $(\mathrm{Ni})$ because they form metal carbides together with carbon precursors which become the seeds for carbon nanotubes growth. Therefore, they often remain built into the tubes and become an integral part of the nanomaterial.

So far, it has been reported that the purification of contaminated CNTs could be achieved via the functionalization of carbon nanotubes [9]. Through this procedure, it is possible to cover the CNTs surface by various chemical groups such as: $-\mathrm{OH},-\mathrm{COOH}, \mathrm{COONH}_{4}$, etc. Furthermore, it has been proven that the traces of catalyst are gradually removed from the nanotubes at the same time [10].

Another material which is interesting in the case of this work is hematite $\left(\alpha-\mathrm{Fe}_{2} \mathrm{O}_{3}\right)$. This oxide belongs to the group of low-cost and environmentally-friendly transition metal 
Fig. 1 TEM images of MWCNTs-COONH 4 (a), multiwall carbon nanotube filled by the trace of catalyst (b), and MWCNTs-COONH 4 coated randomly by $\alpha-\mathrm{Fe}_{2} \mathrm{O}_{3}$ (c)

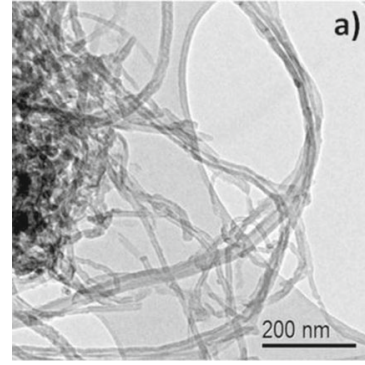

a)
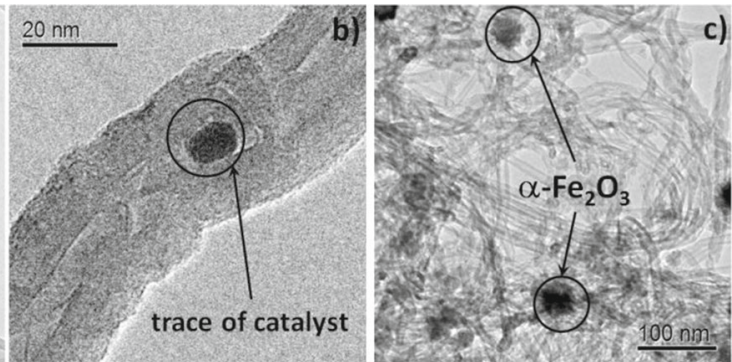

oxides. Moreover, $\alpha-\mathrm{Fe}_{2} \mathrm{O}_{3}$ is the most thermodynamically stable iron oxide and it is extensively used in the production of pigments, jewelry, catalysts, sensors, hard and soft magnets, devices for energy storage and conversion, etc. [11].

The purpose of this work is to synthesize and characterize a new nanocomposite which consists of chemicallymodified multiwall carbon nanotubes (MWCNTs) covered by randomly-deposited nanoparticles of hematite $\left(\alpha-\mathrm{Fe}_{2} \mathrm{O}_{3}\right)$. Such nanomaterial promises to combine the features of both MWCNTs and $\alpha-\mathrm{Fe}_{2} \mathrm{O}_{3}$ making it an useful material in many fields for future applications.

\section{Experimental Details}

The raw multiwall carbon nanotubes (MWCNTs; $93 \%$ of purity; CNT CO., Ltd. from South Korea) were manufactured via CVD process with iron $(\mathrm{Fe})$ as the catalyst. Such MWCNTs were chemically-modified by heating at $120{ }^{\circ} \mathrm{C}$ in concentrated nitric acid $(68 \%$ $\mathrm{HNO}_{3}$ ) for $50 \mathrm{~h}$ under a reflux. Then, they were filtered and washed with distilled water until the $\mathrm{pH}$ value of the filtrate reached 6 . Thereby, the MWCNTs were

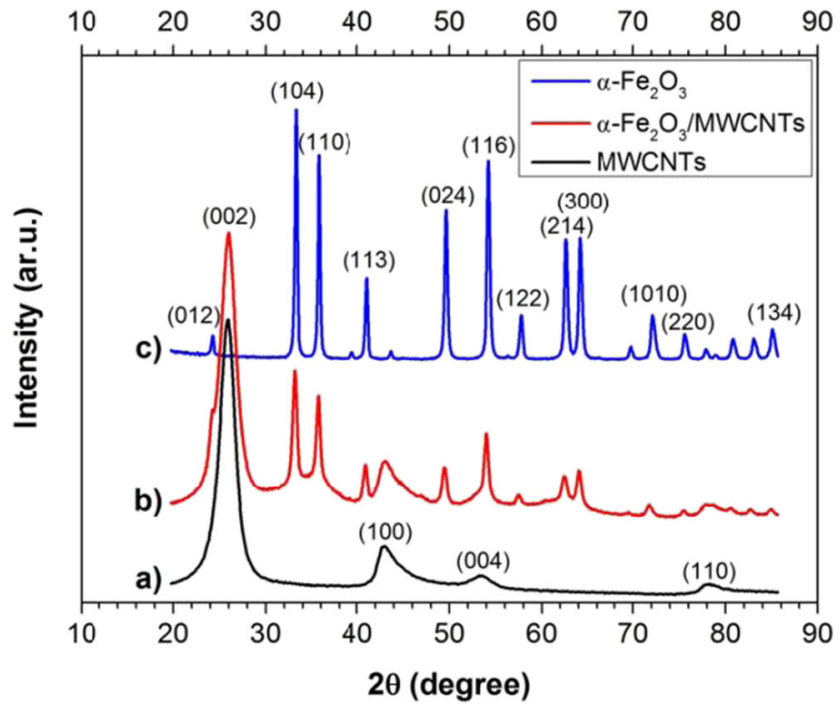

Fig. 2 XRD patterns of MWCNTs-COONH 4 (a), $\alpha-\mathrm{Fe}_{2} \mathrm{O}_{3} /$ MWCNTs (b), and commercial $\alpha-\mathrm{Fe}_{2} \mathrm{O}_{3}$ (c) oxidized and formed carboxylic groups attached to the ends and the walls of CNTs (MWCNTs-COOH) [12]. Such functionalized CNTs were treated with a $25 \%$ aqueous solution of ammonia and then rinsed with distilled water during filtration to obtain the ammonium salt (MWCNTs$\mathrm{COONH}_{4}$ ). After that, $1 \mathrm{~g}$ of MWCNTs-COONH 4 was dispersed in $500 \mathrm{ml}$ of distilled water and cooled to $4{ }^{\circ} \mathrm{C}$. In two other beakers $0.215 \mathrm{~g}$ of iron dichloride tetrahydrate $\left(\mathrm{FeCl}_{2} \cdot 4 \mathrm{H}_{2} \mathrm{O} ; 98 \%\right.$ of purity; Sigma-Aldrich) and $0.588 \mathrm{~g}$ of iron trichloride hexahydrate $\left(\mathrm{FeCl}_{3} \cdot 6 \mathrm{H}_{2} \mathrm{O} ; 97 \%\right.$ of purity; Sigma-Aldrich) were dissolved in distilled water and added by droplets to the previously prepared carbon nanotube dispersion mixed with a magnetic stirrer. After $30 \mathrm{~min}$, the $\mathrm{pH}$ of dispersion was brought up to 10 with $1 \mathrm{M}$ $\mathrm{NaOH}$. The obtained product was heated up to $100{ }^{\circ} \mathrm{C}$ on a hot plate with magnetic stirring for $30 \mathrm{~min}$. Finally, the prepared nanocomposite was centrifuged and washed with distilled water until the value of $\mathrm{pH}$ reached 7.

The obtained nanomaterial was studied using a Phillips $\mathrm{X}$ 'Pert diffractometer (XRD) equipped with a $\mathrm{Cu} \mathrm{X}$-ray source and a parallel beam Bragg reflection mirror, a JEOL JEM 3010 transmission electron microscope (TEM) and an Oxford Instruments Ltd. vibrating sample magnetometer (VSM) in the range of magnetic field between $-0.6 \mathrm{~T}$ and

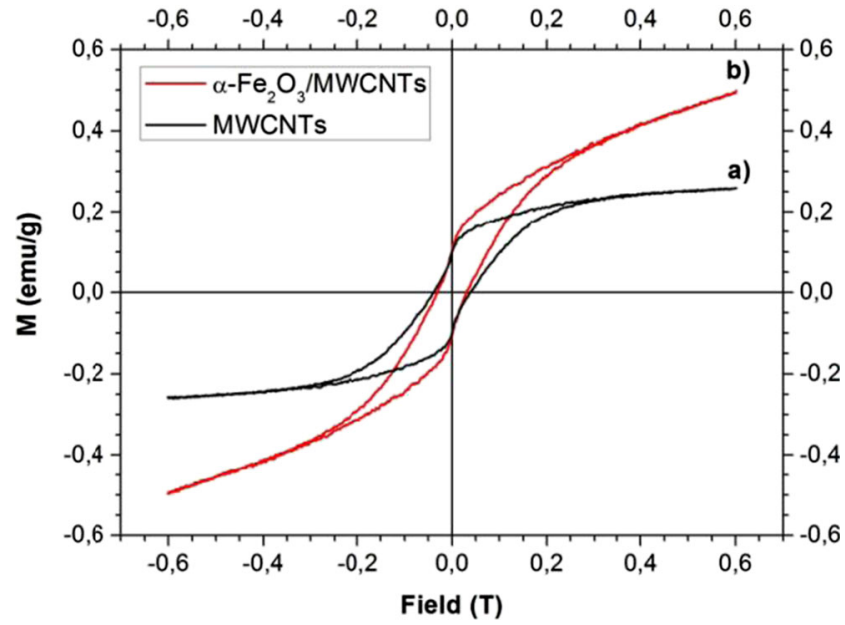

Fig. 3 Magnetization hysteresis loops of MWCNTs-COONH 4 (a) and $\alpha-\mathrm{Fe}_{2} \mathrm{O}_{3} /$ MWCNTs (b) 
0.6 T. The XRD and VSM measurements were performed under ambient conditions.

Additionally, the results for commercially-available reference samples of $\alpha-\mathrm{Fe}_{2} \mathrm{O}_{3}$ powder (99.98\% of purity; Carl Roth $\mathrm{GmbH}$ ) and the chemically-modified multiwall carbon nanotubes (MWCNTs-COONH 4 ) are presented in this work.

\section{Results and Discussion}

TEM images of chemically-modified MWCNTs and $\alpha-\mathrm{Fe}_{2} \mathrm{O}_{3} / \mathrm{MWCNT}$ s nanocomposite are presented in Fig. 1a and 1c. A residual amount of catalyst coming from the preparation process can also be seen built into the nanotube (Fig. 1b). TEM studies show that MWCNTs are very disordered, braided, and are successfully coated by the randomly-dispersed hematite nanoparticles. The diameter of CNTs varies from 10 to $40 \mathrm{~nm}$. The average diameters of $\alpha$ $\mathrm{Fe}_{2} \mathrm{O}_{3}$ and traces of catalyst are approximately $50 \mathrm{~nm}$ and $12 \mathrm{~nm}$, respectively.

XRD has great advantages in comparison with the other experimental techniques because it is simple in terms of implementation and it also has the ability of measuring for a long period of time without any negative effects on the sample. Therefore, the XRD technique is a good tool to study the structure of $\alpha-\mathrm{Fe}_{2} \mathrm{O}_{3} / \mathrm{MWCNT}$ nanocomposite and reference samples. These results are presented in Fig. 2. Recorded XRD patterns confirm that MWCNTs are successfully coated by hematite. The positions of peaks originating from $\alpha-\mathrm{Fe}_{2} \mathrm{O}_{3}$ and the chemicallymodified MWCNTs lie at the same angle positions for all structures. Moreover, no significant changes in the functionalized MWCNTs structure after deposition of hematite are observed.

Figure 3 presents the magnetization hysteresis loops of MWCNTs-COONH 4 and $\alpha-\mathrm{Fe}_{2} \mathrm{O}_{3} / \mathrm{MWCNTs}$. The differences in curve shapes indicate different magnetic behaviors. The functionalized MWCNTs contain the encapsulated traces of catalyst coming from the CVD process. The previous studies performed on this nanomaterial have shown that the residual amount of catalyst is associated with the presence of non-stoichiometric iron carbide $\left(\mathrm{Fe}_{x} \mathrm{C}\right), \alpha-\mathrm{Fe}$ and ferrihydrite [10]. Most of these iron compounds are ferromagnetic, therefore magnetization hysteresis of MWCNTs$\mathrm{COONH}_{4}$ reveals the ferromagnetic behavior. On the other hand, the shape of the magnetization curve for the studied nanocomposite exhibits more complex behavior with a coercivity and large high field susceptibility. It is related to the strong influence of $\alpha-\mathrm{Fe}_{2} \mathrm{O}_{3}$ deposited on the surface of carbon nanotubes being superimposed on the ferromagnetic behavior of the catalyst residue, of which the content in the investigated material is much lower than hematite. The recorded values of saturated magnetization $\left(M_{\mathrm{S}}\right)$, remnant magnetization $\left(M_{\mathrm{R}}\right)$ and coercivity $\left(H_{\mathrm{C}}\right)$ for MWCNTs$\mathrm{COONH}_{4}$ are equal $0.26 \mathrm{emu} / \mathrm{g}, 0.10 \mathrm{emu} / \mathrm{g}$ and $0.04 \mathrm{~T}$ (400 Oe), respectively. For $\alpha-\mathrm{Fe}_{2} \mathrm{O}_{3} / \mathrm{MWCNTs,} \mathrm{the} \mathrm{rem-}$ nant magnetization equals $0.11 \mathrm{emu} / \mathrm{g}$ and the coercivity equals $0.03 \mathrm{~T}$ (300 Oe). Taking into account that all magnetic moment values are given in this work per unit of total mass (emu/g), considering all compounds inside and outside of CNTs and also the nanotubes mass, it is very difficult to perform further analysis of the obtained results. Nevertheless, the presence of encapsulated catalyst traces in CNTs and diamagnetic contribution of the graphitic nanotubes play important roles for the magnetic properties of both MWCNTs-COONH 4 and $\alpha-\mathrm{Fe}_{2} \mathrm{O}_{3}$ /MWCNTs.

\section{Conclusion}

This work confirms that $\alpha-\mathrm{Fe}_{2} \mathrm{O}_{3}$ /MWCNTs nanocomposite could be simply prepared via the proposed chemical method. Morphology and structural and magnetic features of this material were determined. Moreover, the investigated nanomaterial is interesting due to the variety of possible applications; for example in the lithium-ion batteries [4, 13] and/or the supercapacitors [5, 14]. Therefore, it is supposed to be a very perspective material with regards to future research.

Acknowledgments This work was supported by the Foundation for Polish Science International PhD Projects Programme co-financed by the EU European Regional Development Fund, A.M. thanked the support from the European Union funds by the European Social Fund and L.S. thanked the National Center for Research and Development for support by the project no. PBS1/A5/15/2012. Sincere thanks towards Dr. J. Syzdek for the research consultations.

Open Access This article is distributed under the terms of the Creative Commons Attribution License which permits any use, distribution, and reproduction in any medium, provided the original author(s) and the source are credited.

\section{References}

1. Iijima, S.: Nature 354, 56-58 (1991)

2. Liu, Z., Chen, K., Davis, C., Sherlock, S., Cao, Q., Chen, X., Dai, H.: Cancer Res. 68, 6652-6660 (2008)

3. Li, Y.H., Wang, S.G., Zhang, X.F., Wei, J.Q., Xu, C., Luan, Z.K., Wu, D.H.: Mater. Res. Bull. 38, 469-476 (2003)

4. de las Casas, C., Li, W.Z.: J. Power Sources 208, 74-85 (2012)

5. Pan, H., Li, J., Feng, Y.: Nanoscale Res. Lett. 5, 654-668 (2010)

6. Zhang, Y., Gu, H., Iijima, S.: Appl. Phys. Lett. 73, 3827-3829 (1998) 
7. Kong, J., Soh, H.T., Cassell, A.M.: Nature 395, 878-881 (1998)

8. Kukovitsky, E.F., L'vov, S.G., Sainov, N.A.: Chem. Phys. Lett. 317, 65-70 (2000)

9. Jamrozik, A., Mazurkiewicz, M., Malolepszy, A., Stobinski, L., Matlak, K., Korecki, J., Kurzydłowski, K.J., Burda, K.: Phys. Status Solidi A-Appl. Mat. 208, 1783-1786 (2011)

10. Kuryliszyn-Kudelska, I., Małolepszy, A., Mazurkiewicz, M., Stobinski, L., Kurzydłowski, K.J., Dobrowolski, W.: Phys. Status Solidi A-Appl. Mat. 208, 1787-1790 (2011)
11. Cornell, R.M., Schwertmann, U.: The iron oxides. structure, properties, reactions, occurrences and uses: structure, properties, reactions, occurrences and uses. Wiley-VCH, Weinheim (2003)

12. Stobinski, L., Lesiak, B., Kover, L., Toth, J., Biniak, S., Trykowski, G., Judek, J.: J. Alloys Compd. 501, 77-84 (2010)

13. Hanga, B.T., Watanabe, I., Doi, T., Okada, S., Yamaki, J.I.: J. Power Sources 161, 1281-1287 (2006)

14. Xie, K.Y., Li, J., Lai, Y.Q., Lu, W., Zhang, Z., Liu. Y.X., Zhou, L.M., Huang, H.T.: Electrochem. Commun. 13, 657$660(2011)$ 